\title{
Equation of State for a Perfect Solid and Its Application to the Solid-Gas Equilibrium of Argon
}

\author{
Yosuke KATAOKA* and Yuri YAMADA
}
Department of Chemical Science and Technology, Faculty of Bioscience and Applied Chemistry, Hosei University, 3-7-2 Kajino-cho, Koganei, Tokyo 184-8584, Japan
*e-mail: kataoka@hosei.ac.jp

(Received: May 6, 2011; Accepted for publication: November 28, 2011; Advance publication: December 21, 2011)

An equation of state for a perfect solid is proposed, where the system includes only single spherical molecules. A Lennard-Jones interaction is assumed in the nearest neighbors on the face-centered cubic (FCC) lattice. The internal energy is the sum of the average kinetic energy and the potential energy at $0 \mathrm{~K}$, which is a function of the volume. The pressure satisfies the thermodynamic conditions for the internal energy and pressure. The equilibrium condition is solved numerically for the solid-gas equilibrium of argon. The Gibbs energy gives a reasonable vapor pressure for solid argon, if the temperature dependence of the virial term is taken into account.

Keywords: Equation of state, Perfect solid, Sublimation vapor pressure

\section{Introduction}

A perfect gas is one of the most important models in physical chemistry [1]. The equation of state (EOS) for a perfect gas is based on thermodynamics. The solid phase is another limit in matter, where the molecular interaction energy is larger than the kinetic energy. An idealized model is assumed for a system of single spherical molecules at low temperatures. A LennardJones interaction is effective between nearest neighbors in a face-centered cubic (FCC) lattice with volume $V$. The potential energy of a molecular system with $N$ spherical molecules $E_{\mathrm{p}}(V$, $0 \mathrm{~K}$ ), is evaluated at $0 \mathrm{~K}$ using classical mechanics. The uniform expansion to a very large volume will be allowed by the same function, $E_{\mathrm{p}}(V, 0 \mathrm{~K})$.

The internal energies of a perfect solid $U$ are the sum of the thermal average of the kinetic and potential energies. The pressure $p$ must satisfy the thermodynamic conditions for the internal energy and pressure [1]:

$$
\left(\frac{\partial U}{\partial V}\right)_{T}=T\left(\frac{\partial p}{\partial T}\right)_{V}-p .
$$

A solution for the pressure is obtained, so that the internal energy EOS and pressure EOS can be given by simple analytical expressions in terms of $V$ and the temperature $T$.
Changes in the entropy $\Delta S$ are calculated using the EOS according to isothermal reversible expansion and heating at constant volume. A reasonable origin of the entropy $S_{0}$ will be defined for a very large volume and typical temperature. It will be shown that the same origin can be selected for a perfect gas EOS.

The enthalpy $H$, the Helmholtz energy $A$, and the Gibbs energy $G$ are defined by standard methods [1]. The equilibrium condition is solved graphically for the solid-gas equilibrium of argon. The Gibbs energy will provide a reasonable vapor pressure for solid argon [2] if the temperature dependence of the virial term is taken into account with respect to the pressure.

\section{Potential energy at $0 \mathrm{~K}$}

Molecular interaction of the Lennard-Jones form is assumed. The Lennard-Jones potential $u(r)$, is expressed as a function of the interatomic distance, $r$ :

$$
u(r)=4 \varepsilon\left[\left(\frac{\sigma}{r}\right)^{12}-\left(\frac{\sigma}{r}\right)^{6}\right]
$$

where $\varepsilon$ is the depth of the potential well, and $\sigma$ is the separation at which $u(\sigma)=0$. This function has the minimum value: 


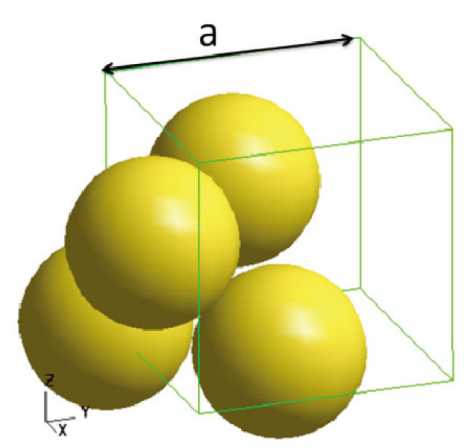

Figure 1. FCC structure and the lattice constant $a$.

$u\left(r_{0}\right)=-\varepsilon$

at a distance

$$
r_{0}=2^{1 / 6} \sigma
$$

The molecules have a stable FCC structure based on this interaction (see Figure 1). The sum of $u\left(r_{0}\right)$ for nearest neighbors is given as:

$$
\sum_{i=1}^{12} u\left(r_{0}\right)=-12 \varepsilon
$$

where the optimized crystal structure with $N$ molecules has the following minimum potential energy at $0 \mathrm{~K}$ :

$$
E_{\mathrm{pmin}}=6 N u\left(r_{0}\right)=-6 \varepsilon N \text {. }
$$

The coefficient is 6 and not 12, because every site is equivalent and the interaction energy must be counted only once.

For the general volume $V$, the potential energy at $0 \mathrm{~K}$ is calculated in the following way. The lattice constant $a$ is defined in Figure 1, and there are four molecules in the cube. The volume per molecule is

$$
\frac{V}{N}=\frac{a^{3}}{4}
$$

The lattice constant and the nearest neighbor distance $r_{\mathrm{n}}$ have the following relation:

$$
\frac{a}{\sqrt{2}}=r_{\mathrm{n}} .
$$

$r_{\mathrm{n}}$ is then expressed by the volume per molecule, $V / N$ :

$$
r_{\mathrm{n}}=\left(\sqrt{2} \frac{V}{N}\right)^{1 / 3}
$$

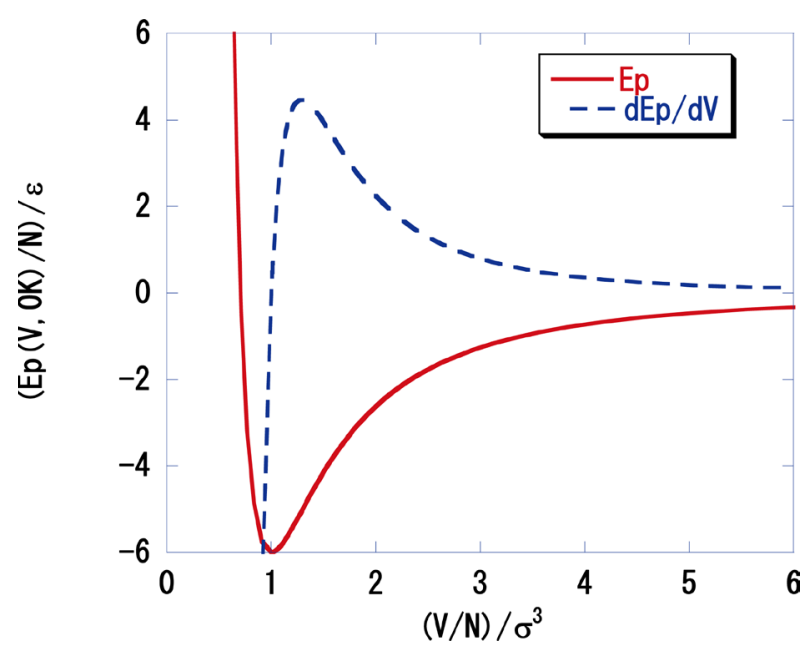

Figure 2. Potential energy at $0 \mathrm{~K}, E_{\mathrm{p}}(V, 0 \mathrm{~K})$, and its derivative with respect to the volume $V v s$. the volume per molecule $V / N$.

Substituting this for eq (6) gives the potential energy of a system with volume $V$ at $0 \mathrm{~K}$ :

$$
\frac{E_{\mathrm{p}}(V, 0 K)}{\varepsilon N}=12\left[\frac{1}{2}\left(\frac{\sigma^{3}}{v}\right)^{4}-\left(\frac{\sigma^{3}}{v}\right)^{2}\right], \quad v \equiv \frac{V}{N} .
$$

The potential energy at $0 \mathrm{~K}$ is reduced by the energy constant $\varepsilon$, and can be plotted as a function of the volume per molecule reduced by the unit volume $\sigma^{3}$, as shown in Figure 2. This gives the minimum value $-6 \varepsilon N$ at $v=\sigma^{3}$.

\section{Internal energy}

The average kinetic energy is given as:

$$
\left\langle E_{\mathrm{k}}\right\rangle=\frac{3}{2} N k T
$$

where $k$ is the Boltzmann constant. The internal energy of a perfect solid $U$ is the sum of the thermal average of the kinetic energy and the potential energy:

$$
U(V, T)=\frac{3}{2} N k T+E_{\mathrm{p}}(V, 0 \mathrm{~K}) .
$$

This is the primitive internal energy EOS for a perfect solid. The explicit expression for the second term on the right hand side is given in eq (10).

\section{Pressure}

The pressure and internal energy must satisfy the thermodynamic condition given in eq (1), which is derived from the fun- 


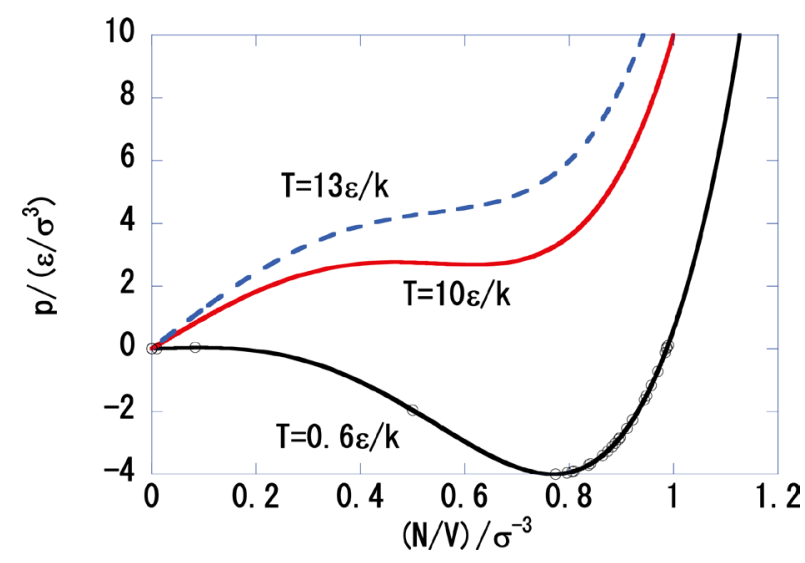

Figure 3. Pressure $p v s$. the number density $N / V$ at several temperatures.

damental equation of thermodynamics [1]. By substitution of eq (12) for eq (1), the following equation is obtained:

$$
\left(\frac{\partial E_{\mathrm{p}}(V, 0 \mathrm{~K})}{\partial V}\right)_{T}=T\left(\frac{\partial p}{\partial T}\right)_{V}-p .
$$

One simple solution for eq (13) is given as:

$$
p(V, T)=\frac{N k T}{V}-\left(\frac{\partial E_{\mathrm{p}}(V, 0 \mathrm{~K})}{\partial V}\right)_{T} .
$$

This can be confirmed by the substitution of eq (14) for the right hand side of eq (13). This is the primitive pressure EOS for a perfect solid.

The derivative of the potential energy with respect to the volume is given as:

$$
\left(\frac{\partial E_{\mathrm{p}}(V, 0 \mathrm{~K})}{\partial V}\right)_{T}=-24 \frac{\varepsilon N}{\sigma^{3}}\left[\frac{1}{\left(\frac{V}{\sigma^{3} N}\right)^{5}}-\frac{1}{\left(\frac{V}{\sigma^{3} N}\right)^{3}}\right] .
$$

This quantity is shown as a function of the reduced volume $(V / N) / \sigma^{3}$, in Figure 2. The pressure is plotted at several temperatures in Figure 3. The curves are similar to those for the van der Waals EOS [1]. Only the state at low temperature and with volume close to $V / N=\sigma^{3}$ represents the solid state; the other states are extrapolated. In the low density limit, the pressure tends toward that of the perfect gas EOS. The low density state is used to fix the origin of the entropy.

\section{Reversible isothermal expansion}

The heat obtained by a perfect solid during reversible isother-

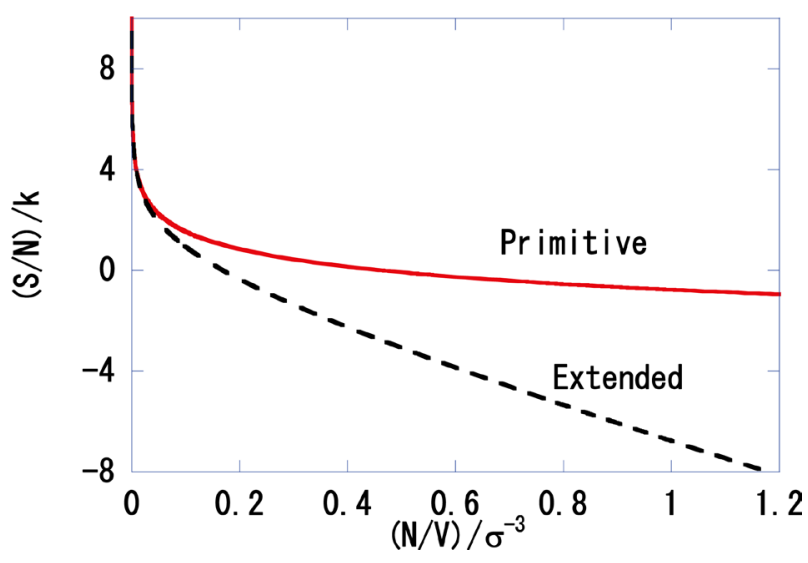

Figure 4. The entropy per molecule $S / N v s$. the number density $N / V$, at $T=0.6 \varepsilon / k$.

mal expansion is calculated using the internal energy EOS and the pressure EOS. The work in this process, from the initial volume $V_{\mathrm{i}}$, to the final volume $V_{\mathrm{f}}$, is given by this formula [1]:

$$
w=-\int_{V_{\mathrm{i}}}^{V_{\mathrm{f}}} p \mathrm{~d} V
$$

By substitution of $p$ in eq (16) for this right hand side, the work done by the perfect solid $w$ is given as:

$$
w=-N k T \ln \frac{V_{\mathrm{f}}}{V_{\mathrm{i}}}+E_{\mathrm{p}}\left(V_{\mathrm{f}}, 0 \mathrm{~K}\right)-E_{\mathrm{p}}\left(V_{\mathrm{i}}, 0 \mathrm{~K}\right) .
$$

The change in the internal energy is expressed as:

$$
\Delta U=E_{\mathrm{p}}\left(V_{\mathrm{f}}, 0 \mathrm{~K}\right)-E_{\mathrm{p}}\left(V_{\mathrm{i}}, 0 \mathrm{~K}\right) .
$$

The heat for the perfect solid $q$, is then calculated by the first law of thermodynamics:

$$
q=\Delta U-w=N k T \ln \frac{V_{\mathrm{f}}}{V_{\mathrm{i}}} .
$$

This heat is used to obtain $\Delta S$ by reversible isothermal expansion.

\section{Heating process at constant volume}

Let us consider that the state is changed from $\left(T_{\mathrm{i}}, V\right)$ to $\left(T_{\mathrm{f}}, V\right)$ by the heating process at a constant volume $V$. The heat capacity at a constant volume of the perfect solid $C_{V}$ is given by the internal energy EOS in eq (12).

$$
C_{V}=\frac{3}{2} N k
$$

The heat $q$ and work $w$ obtained for the perfect solid in this 


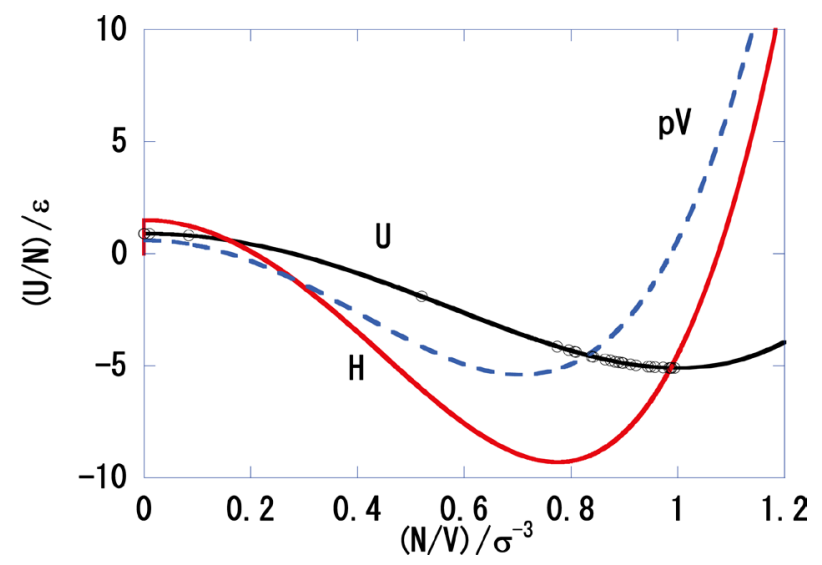

Figure 5. Enthalpy per molecule $H / N$ vs. the number density $N / V$, at $T=0.6 \varepsilon / k$.

process are given as:

$$
q=C_{V} \Delta T=C_{V}\left(T_{\mathrm{f}}-T_{\mathrm{i}}\right), \quad w=0 .
$$

The heat is used in the next section to calculate $\Delta S$ by the heating process at constant volume $V$.

\section{Volume-and temperature-dependence of entropy}

The state is changed from $\left(T_{\mathrm{i}}, V_{\mathrm{i}}\right)$ to $\left(T_{\mathrm{f}}, V_{\mathrm{i}}\right)$ by the heating process at constant volume $V_{\mathrm{i}}$; then, isothermal reversible expansion from the initial volume $V_{\mathrm{i}}$ to the final volume $V_{\mathrm{f}}$ is considered at temperature $T_{\mathrm{f}}$. From the previous results, it can be shown that the entropy $S$ is dependent on the volume and the temperature as [1]:

$$
\begin{aligned}
\Delta S & =N k \ln \left(\frac{V_{\mathrm{f}}}{V_{\mathrm{i}}}\right)+\int_{T_{\mathrm{i}}}^{T_{\mathrm{f}}} C_{V} \frac{\mathrm{d} T}{T} \\
& =N k \ln \left(\frac{V_{\mathrm{f}}}{V_{\mathrm{i}}}\right)+\frac{3}{2} N k \ln \left(\frac{T_{\mathrm{f}}}{T_{\mathrm{i}}}\right) .
\end{aligned}
$$

A state with a sufficiently large volume $N \boldsymbol{v}_{\max }$ and a typical temperature $\varepsilon / k$, is selected as a standard state to define the origin of the entropy of the system:

$$
\begin{aligned}
& \Delta S=S(V, T)-S\left(N v_{\max }, \varepsilon / k\right) \\
& =N k \ln \left(\frac{V}{N v_{\text {max }}}\right)+\frac{3}{2} N k \ln \left(\frac{T}{\varepsilon / k}\right) \\
& =N k \ln \left(\frac{V}{\sigma^{3} N}\right)+\frac{3}{2} N k \ln \left(\frac{T}{\varepsilon / k}\right)-N k \ln \left(\frac{N v_{\max }}{\sigma^{3} N}\right) \\
& \equiv N k \ln \left(\frac{V}{\sigma^{3} N}\right)+\frac{3}{2} N k \ln \left(\frac{T}{\varepsilon / k}\right)-S_{0} .
\end{aligned}
$$

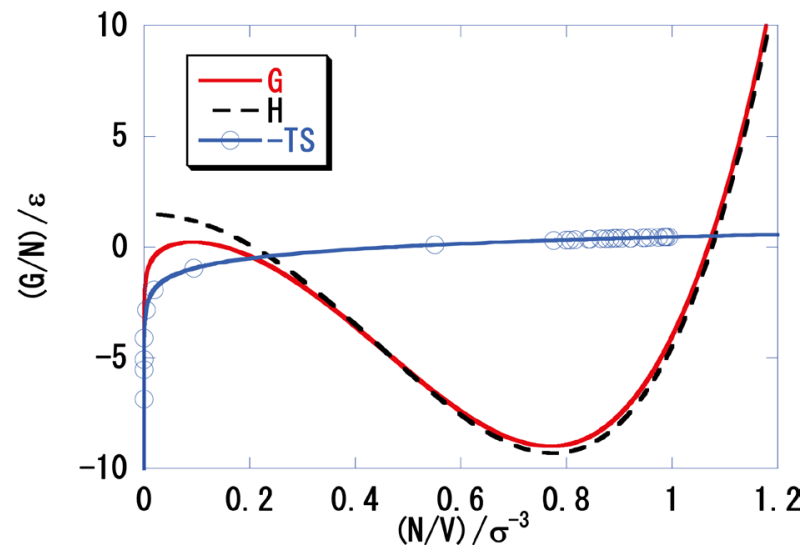

Figure 6. Gibbs energy per molecule $G / N$ vs. the number density $N / V$, at $T=0.6 \varepsilon / k$.

Here, the origin of the entropy $S_{0}$ is defined as:

$$
S_{0} \equiv S\left(N v_{\max }, \varepsilon / k\right) \equiv N k \ln \left(\frac{\boldsymbol{v}_{\max }}{\sigma^{3}}\right) .
$$

This value is the origin of the entropy in the perfect solid; therefore, the entropy is expressed as:

$$
S(V, T)=N k \ln \left(\frac{V}{\sigma^{3} N}\right)+\frac{3}{2} N k \ln \left(\frac{T}{\varepsilon / k}\right) .
$$

The origin of the entropy in a perfect gas is assumed in the same way, so that the entropy of a perfect gas $S_{\mathrm{g}}$ is given as [1]:

$$
S_{\mathrm{g}}(V, T)=N k \ln \left(\frac{V}{\sigma^{3} N}\right)+\frac{3}{2} N k \ln \left(\frac{T}{\varepsilon / k}\right) .
$$

The entropy $S$ and that for the extended model $S_{\text {ext }}$, which is introduced in the final section, are shown as functions of the number density at $T=0.6 \varepsilon / k$ in Figure 4 .

\section{Gibbs energy}

The enthalpy $H$, Helmholtz energy $A$ and Gibbs energy $G$ are defined as:

$$
\begin{aligned}
& H=U+p V, \\
& A=U-T S, \\
& G=H-T S .
\end{aligned}
$$

The enthalpy and Gibbs energy are plotted as functions of the number density in Figures 5 and 6. The internal energy is also shown for comparison in Figure 5. The $p V$ term is dominant in 


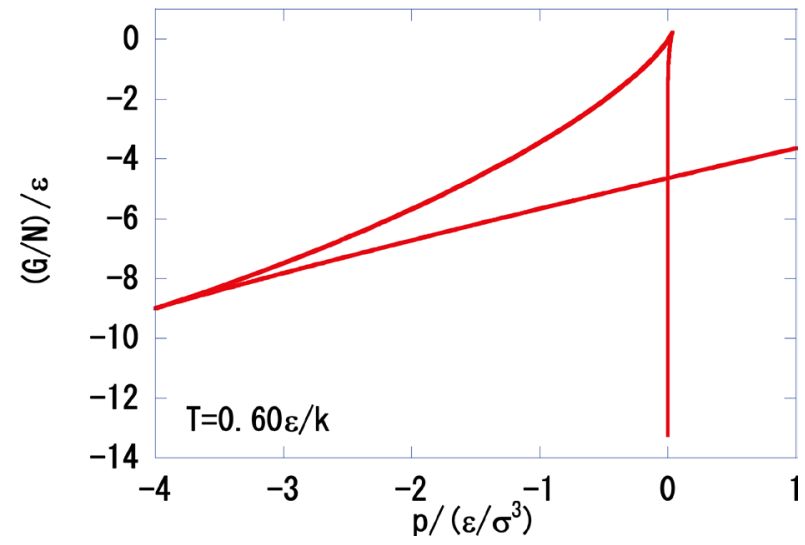

Figure 7. Entire Gibbs energy vs. the pressure plot at $T=0.6$ $\varepsilon / k$.

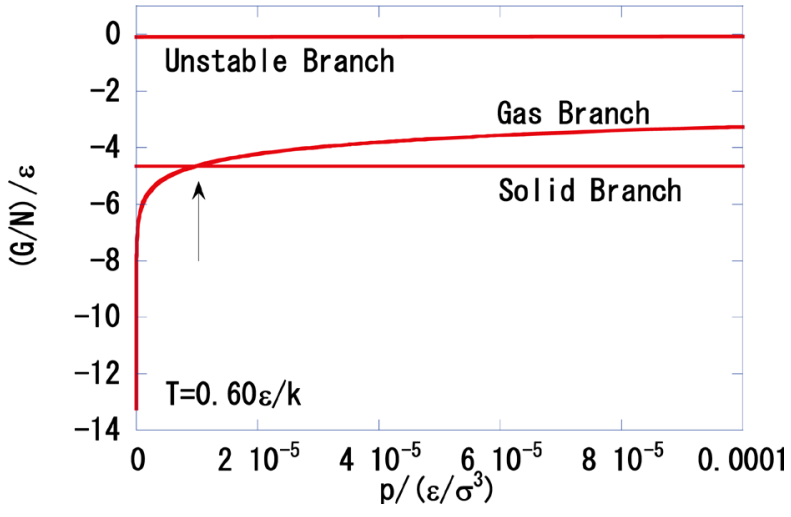

Figure 8. Gibbs energy per molecule $G / N v s$. the pressure $p$, at $T=0.6 \varepsilon / k$, showing solid and gas branches. The arrow indicates the solid-gas equilibrium.

eq (27) around $(V / N)=\sigma^{3}$. The Gibbs energy is compared with the entropy term $-T S$ in Figure 6. The variation of the Gibbs energy comes from the $-T S$ term at large volume and from the $p V$ term in the case of a small volume.

\section{Solid-gas equilibrium}

The solid-gas equilibrium is considered by the pressure EOS and the Gibbs energy. The following equations must be solved:

$$
p\left(V_{\mathrm{s}}, T\right)=p_{\mathrm{g}}\left(V_{\mathrm{g}}, T\right), \quad G\left(V_{\mathrm{s}}, T\right)=G_{\mathrm{g}}\left(V_{\mathrm{g}}, T\right) .
$$

The variables to be obtained are $V_{\mathrm{s}}, V_{\mathrm{g}}$ and $T$. The temperature $T$ is selected first. The equations are solved graphically, as shown in Figures 7 and 8. Firstly the Gibbs energy for a perfect solid $G$, and the pressure $p$, are tabulated as functions of the volume at the given $T$. The Gibbs energy is plotted as a function of the

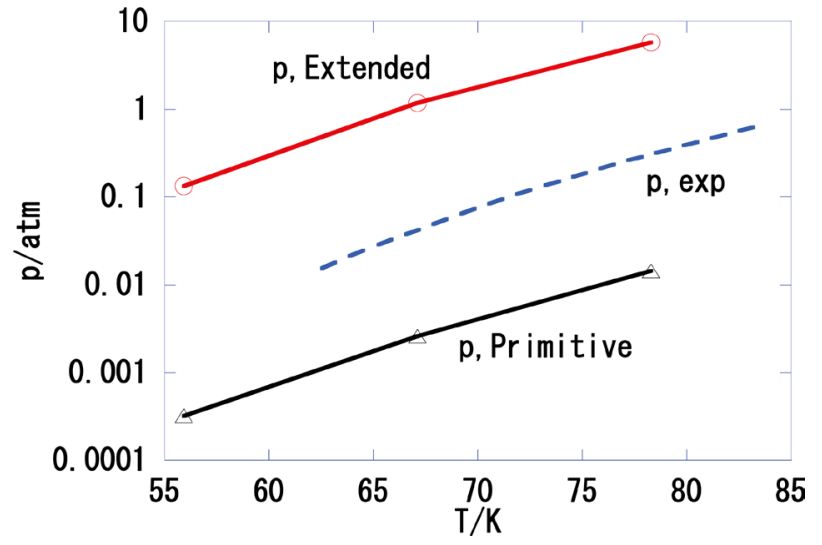

Figure 9. Comparison of the vapor pressure of solid argon with experimental vapor pressure [2].

Table 1. Lennard-Jones parameters for argon [1].

$1 \mathrm{~atm}=101325 \mathrm{~Pa}$.

\begin{tabular}{ccccc}
\hline$(\varepsilon / k) / \mathrm{K}$ & $\varepsilon / \mathrm{J}$ & $\sigma / \mathrm{m}$ & $\left(\varepsilon / \sigma^{3}\right) / \mathrm{Pa}$ & $\left(\varepsilon / \sigma^{3}\right) / \mathrm{atm}$ \\
\hline 111.84 & $1.54 \mathrm{E}-21$ & $3.623 \mathrm{E}-10$ & $3.25 \mathrm{E}+07$ & $3.20 \mathrm{E}+02$ \\
\hline
\end{tabular}

pressure, where the volume acts as an auxiliary variable. The solid and gas branches are shown in the entire $G$ - $p$ plot in Figure 7. The crossing point in the $G$ vs. $p$ curve is the solution to eq (30). The region of the crossing point is shown in Figure 8. The almost horizontal solid branch line in Figure 8 is the Gibbs energy for a perfect solid.

The solid-gas equilibrium state is obtained as follows:

$$
\begin{aligned}
& T=0.6 \varepsilon k^{-1}, \quad p=9.9 \times 10^{-6} \varepsilon \sigma^{-3}, \\
& G / N=-4.6 \varepsilon .
\end{aligned}
$$

The solutions for the volume of each phase are obtained from the pressure EOS:

$$
V_{\mathrm{s}} / N=1.01 \sigma^{3}, \quad V_{\mathrm{g}} / N=6.1 \times 10^{4} \sigma^{3} .
$$

\section{Vapor pressure of solid argon}

The vapor pressure of solid argon is calculated based on the Lennard-Jones parameters [1] given in Table 1. The vapor pressure obtained is compared with the experimental results [2] in Figure 9. The curve $p$, primitive indicates the phase equilibrium between a perfect solid (solid branch) and a perfect gas (gas branch). Although the calculated values are smaller than the experimental values over the entire temperature region, the order of magnitude is not far from that observed. In this sense, this simple model represents the most important feature of the solid- 


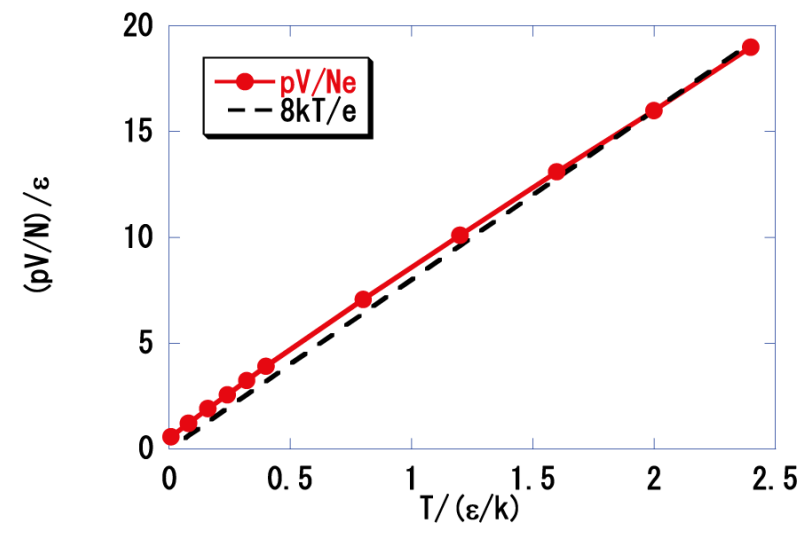

Figure 10. Pressure vs. temperature for solid argon by NTV molecular dynamics simulation. The number of molecules in the basic cell is 256 . The volume per molecule is $0.915 \sigma^{3}$. The cut-off distance is $29 \sigma$.

gas equilibrium. In order to obtain better agreement with the experimental results, we performed thermal averaging of the virial pressure term by the harmonic oscillator approximation; the results are shown in the next section.

Other thermodynamic quantities, such as isothermal compressibility, the thermal expansion coefficient, and the heat capacity at constant pressure are obtained by standard methods [1].

\section{An extended EOS for a perfect solid}

We now consider the effect of temperature on the interaction term for the pressure. The pressure is given by the following virial expression [3]:

$$
\begin{aligned}
& p V=N k T+\langle\text { Virial }\rangle, \\
& \langle\text { Virial }\rangle=\langle\text { Virial }\rangle_{T=0 K}+\langle\text { Virial }\rangle_{T>0} .
\end{aligned}
$$

The first term is given by eq (15) and the second term is expressed as [3]:

$$
\begin{aligned}
& \langle\text { Virial }\rangle_{T=0 \mathrm{~K}}=-\left(\frac{\partial E_{\mathrm{p}}(V, 0 \mathrm{~K})}{\partial V}\right)_{T}, \\
& \langle\text { Virial }\rangle_{T>0}=\frac{1}{3}\left\langle\sum_{i}^{N} \mathbf{r}_{i} \cdot \mathbf{f}_{i}\right\rangle_{T>0}=\frac{1}{3}\left\langle\sum_{i}^{N} \sum_{j>i} \mathbf{r}_{i} \cdot \mathbf{f}_{i j}\right\rangle_{T>0} .
\end{aligned}
$$

The force $\mathbf{f}_{i j}$ is the interaction between $i$-th and $j$-th molecules:

$$
\mathbf{r}_{i j}=\mathbf{r}_{j}-\mathbf{r}_{i}, \quad \mathbf{f}_{i j}=-k_{\mathrm{H}} \Delta \mathbf{r}_{i j},
$$

where $k_{\mathrm{H}}$ is the proportionality coefficient of Hooke's law. Here, the position and the potential energy are expanded near the equilibrium site of the $i$-th molecule $\mathbf{r}_{i \mathrm{e}}$

$$
\mathbf{r}_{i}=\mathbf{r}_{i \mathrm{e}}+\Delta \mathbf{r}_{i}, \quad \Delta \mathbf{r}_{i j}=\mathbf{r}_{j}-\mathbf{r}_{i \mathrm{e}}-\Delta \mathbf{r}_{i}
$$

It is assumed that there are 12 nearest neighbor sites. In this way, the temperature effect on the virial term is obtained as follows:

$$
\begin{aligned}
& \frac{1}{3}\left\langle\sum_{i=1}^{N} \sum_{j>i} \mathbf{r}_{i} \cdot \mathbf{f}_{i j}\right\rangle_{T>0 \mathrm{~K}} \\
& =\frac{1}{3}\left\langle\sum_{i=1}^{N} \sum_{j>i}\left(\mathbf{r}_{i \mathrm{e}}+\Delta \mathbf{r}_{i}\right) \cdot\left(-k_{\mathrm{H}}\left(\mathbf{r}_{j}-\mathbf{r}_{i \mathrm{e}}-\Delta \mathbf{r}_{i}\right)\right)\right\rangle_{T>0 \mathrm{~K}} \\
& =2\left\langle\sum_{i=1}^{N}\left(\Delta \mathbf{r}_{i}\right) \cdot\left(k_{\mathrm{H}} \Delta \mathbf{r}_{i}\right)\right\rangle_{T>0 \mathrm{~K}}=2 N \cdot 3 \cdot 2 \cdot \frac{k T}{2}=6 N k T .
\end{aligned}
$$

In the last part, the virial theorem is used [3]. The temperature dependence of the virial term is confirmed by molecular dynamics simulation for solid argon, as shown in Figure 10. The molecular dynamics simulations were performed with a long cut-off distance of $29 \sigma$, so that the temperature effect on the virial term is approximately $8 N k T$. This effect should be taken into account only near the density where $\sigma^{3} / v \approx 1$. For this reason, the right hand side of eq (37) is multiplied with a weight function, $\sigma^{3 / v}$.

Equation (38) is used as the extended pressure EOS for a perfect solid.

$$
p=\frac{N k T}{V}-\left(\frac{\partial E_{\mathrm{p}}(V, 0 \mathrm{~K})}{\partial V}\right)_{T}+\frac{6 \sigma^{3} N k T}{V^{2}} .
$$

The potential energy at $0 \mathrm{~K}$ is also modified to express the low density limit in a better approximation. The following weight functions are assumed in the low and high density regions:

$$
w_{\infty}(v)=1-\frac{\sigma^{3}}{v}, \quad w_{s}(v)=\frac{\sigma^{3}}{v}
$$

The following long- and short-range force effects on the potential energy are assumed:

$$
E_{\infty}(v)=-\frac{4 \varepsilon \sigma^{3} N}{v}, \quad E_{\mathrm{s}}(v)=E_{\mathrm{p}}(V, 0 \mathrm{~K}) .
$$

The long-range effect is the internal energy in the van der Waals EOS [4]. The potential energy in the extended EOS is expressed as:

$$
\begin{aligned}
E_{\mathrm{p}, \mathrm{ext}}(V, 0 \mathrm{~K})= & w_{\infty}(V / N) E_{\infty}(V / N) \\
& +w_{\mathrm{s}}(V / N) E_{\mathrm{s}}(V / N) .
\end{aligned}
$$

This function is plotted in Figure 11, including the first and the second terms on the right hand side. The total extended pressure 


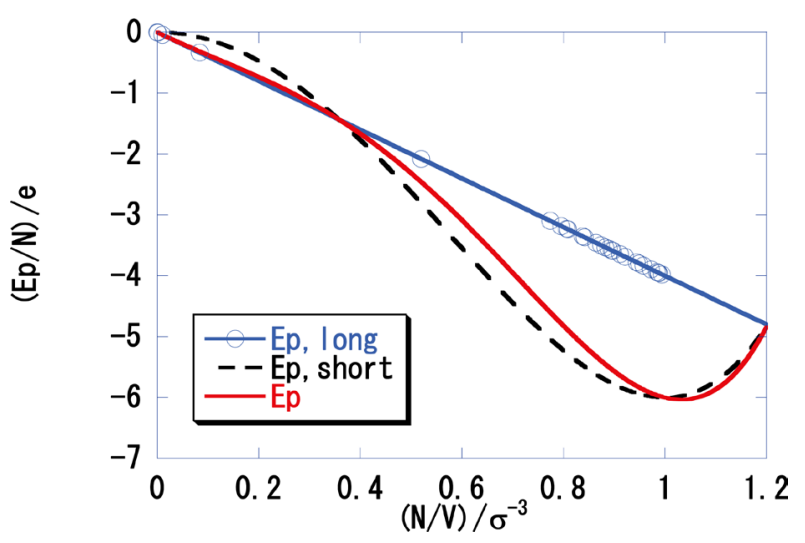

Figure 11. Potential energy of a perfect solid in the extended EOS $v s$. the number density $N / V$.

EOS for a perfect solid is given as:

$$
p(V, T)=\frac{N k T}{V}-\left(\frac{\partial E_{\mathrm{p}, \mathrm{ext}}(V, 0 \mathrm{~K})}{\partial V}\right)_{T}+\frac{6 \sigma^{3} N k T}{V^{2}},
$$

where the work and the heat due to isothermal expansion are given as:

$$
\begin{aligned}
w= & -N k T \ln \frac{V_{\mathrm{f}}}{V_{\mathrm{i}}}+E_{\mathrm{p}}\left(V_{\mathrm{f}}, 0 \mathrm{~K}\right) \\
& -E_{\mathrm{p}}\left(V_{\mathrm{i}}, 0 \mathrm{~K}\right)+6 \sigma^{3} N^{2} k T\left(\frac{1}{V_{\mathrm{f}}}-\frac{1}{V_{\mathrm{i}}}\right)
\end{aligned}
$$

and

$$
\begin{aligned}
q & =\Delta U-w \\
& =N k T \ln \frac{V_{\mathrm{f}}}{V_{\mathrm{i}}}-6 \sigma^{3} N^{2} k T\left(\frac{1}{V_{\mathrm{f}}}-\frac{1}{V_{\mathrm{i}}}\right) .
\end{aligned}
$$

The entropy change is expressed as:

$$
\begin{aligned}
\Delta S & \equiv S\left(V_{\mathrm{f}}, T_{\mathrm{f}}\right)-S\left(V_{i}, T_{\mathrm{i}}\right) \\
& =N k \ln \left(\frac{V_{\mathrm{f}}}{V_{\mathrm{i}}}\right)-6 \sigma^{3} N^{2} k\left(\frac{1}{V_{\mathrm{f}}}-\frac{1}{V_{\mathrm{i}}}\right)+\frac{3}{2} N k \ln \left(\frac{T_{\mathrm{f}}}{T_{\mathrm{i}}}\right) .
\end{aligned}
$$

The start point is then selected as $V_{\mathrm{i}}=N \boldsymbol{v}_{\max }, T_{\mathrm{i}}=\varepsilon / k$ :

$$
\begin{aligned}
\Delta S=S(V, T)-S\left(N v_{\max }, \varepsilon / k\right) \\
=N k \ln \left(\frac{V}{N v_{\max }}\right)-6 \sigma^{3} N^{2} k\left(\frac{1}{V}-\frac{1}{N v_{\max }}\right) \\
\quad+\frac{3}{2} N k \ln \left(\frac{T}{\varepsilon / k}\right) \\
\approx N k \ln \left(\frac{V}{N v_{\max }}\right)-6 \sigma^{3} N^{2} k \frac{1}{V}+\frac{3}{2} N k \ln \left(\frac{T}{\varepsilon / k}\right) \\
=N k \ln \left(\frac{V}{N \sigma^{3}}\right)-6 \sigma^{3} N^{2} k \frac{1}{V}+\frac{3}{2} N k \ln \left(\frac{T}{\varepsilon / k}\right) \\
\quad-N k \ln \left(\frac{N v_{\max }}{\sigma^{3} N}\right) \\
\equiv N k \ln \left(\frac{V}{N \sigma^{3}}\right)-6 \sigma^{3} N^{2} k \frac{1}{V}+\frac{3}{2} N k \ln \left(\frac{T}{\varepsilon / k}\right)-S_{0} .
\end{aligned}
$$

where the constant $S_{0}$ is the same as that given in eq (24). For this reason the following expression is used;

$$
S_{\mathrm{ext}}=N k \ln \left(\frac{V}{\sigma^{3} N}\right)-6 \frac{\sigma^{3} N^{2} k}{V}+\frac{3}{2} N k \ln \left(\frac{T}{\varepsilon / k}\right) \text {. }
$$

The phase equilibrium between solid and gas-phase argon is calculated using the extended EOS for a perfect solid; the results are shown in Figure 10. The vapor pressures calculated by some extension of the EOS for a perfect solid give results that are larger than the experimental results. It is shown that the EOS for a perfect solid gives reasonable vapor pressure values in solid argon.

\section{Problem to be solved in future}

The temperature dependence of the potential energy is not included in the EOS. The energy from the harmonic oscillator approximation is expected to influence the solid branch. No simple solution for the internal energy has yet been found in the consistency of eq (1) for the temperature-dependent potential energy.

This study was supported in part by the Research Center for Computing and Multimedia Studies, Hosei University.

\section{References}

[1] P. W. Atkins, Physical Chemistry, Oxford Univ. Press, Oxford (1998).

[2] The Chemical Society of Japan, Kagaku-Binran Kisohen Kaitei-yonhan, Maruzen, Tokyo (1993).

[3] M. P. Allen, D. J. Tildesley, Computer Simulation of Liquids, Clarendon Press, Oxford (1992).

[4] Y. Kataoka, Y. Yamada, J. Comput. Chem. Jpn, 8, 97-104 (2009). [CrossRef] 